New windows on massive stars: asteroseismology, interferometry, and spectropolarimetry

Proceedings IAU Symposium No. 30\%, 2014

G. Meynet, C. Georgy, J. H. Groh 8 Ph. Stee, eds.

(C) International Astronomical Union 2015 doi:10.1017/S1743921314006413

\title{
Massive OB stars at varying $Z$
}

\author{
A. Herrero ${ }^{1,2}$, M. Garcia ${ }^{3}$, S. Simón-Díaz ${ }^{1,2}$, I. Camacho ${ }^{1,2}$, \\ C. Sabín-Sanjulián ${ }^{4}$ and N. Castro ${ }^{5}$ \\ ${ }^{1}$ Instituto de Astrofísica de Canarias, C/Vía Láctea s/n, E-38200 La Laguna, Spain \\ email: ahd@iac.es \\ ${ }^{2}$ Departamento de Astrofísica, Universidad de La Laguna, La Laguna, Spain \\ ${ }^{3}$ Centro de Astrobiología, Madrid, CSIC, Spain \\ ${ }^{4}$ Departamento de Física, Universidad de La Serena, Chile \\ ${ }^{5}$ Argelander Institut für Astronomie, Auf dem Hügel 71, 53121 Bonn, Germany
}

\begin{abstract}
Massive stars play a key role in environments with very different metallicities. To interpret the role of massive stars in these systems we have to know their properties at different metallicities. The Local Group offers an excellent laboratory to this aim.
\end{abstract}

Keywords. stars: fundamental parameters, supergiants, winds; galaxies: Local Group

\section{Introduction}

Massive stars play a key role in environments with very different metallicities $(Z)$ : (a) in the re-ionization of the early Universe (Robertson et al. 2010); (b) in the formation of violent phenomena (SNe, GRBs, BH, NS, interacting massive binaries; Langer 2012); (c) in the determination of Starburst properties and Star Formation Rates through UV fluxes or $\mathrm{H}_{\alpha}$ emission (Kennicutt \& Evans 2012); or (d) in the chemical evolution of galaxies through stellar winds, supernova explosions and binary interaction. To interpret the role of massive stars in these systems and our observations of starbursts and high-redshift galaxies we have to know their properties at different metallicities. The Local Group offers an excellent laboratory to this aim because we can observe and analyze massive stars individually in varying metallicity conditions. We present here a short summary of our work in some Local Group galaxies, breaking the metallicity frontier of the SMC.

\section{OB stars in galaxies of varying $Z$}

\subsection{Sextans $A\left(Z=0.07 Z_{\odot}\right)$}

Candidate OB stars in Sextans A were selected following the criteria in Garcia \& Herrero (2013) for IC1613. Long-slit spectra were subsequently obtained with OSIRIS@GTC at R $=1000$ spectral power in the wavelength interval between 4000 and $5500 \AA$. Five O stars and eight B stars were observed and classified, and stellar parameters have been obtained for the O stars using the iacob-gbat tool (Simón-Díaz et al. 2011). These constitute the largest atlas of individual OB stars at such low metallicities. Final analyses will be presented in Camacho et al. (2014, in prep).

$$
\text { 2.2. } I C 1613\left(Z=0.13 Z_{\odot}\right)
$$

IC 1613 candidates were selected following the criteria in Garcia \& Herrero (2013). Longslit spectra of IC 1613 OB stars were obtained with OSIRIS@GTC at R = 1000 in the wavelength interval between 4000 and $5500 \AA$ and multi-object spectroscopy was carried out with VIMOS@VLT at $\mathrm{R}=1800$ covering the interval between 4000 and 
$6800 \AA$ (Herrero et al. 2012). 8 O-stars have been observed with GTC and their parameters were determined using the iacob-gbat tool (Simón-Díaz et al. 2011), allowing us to establish the $T_{\text {eff }}$ scale of $\mathrm{O}$ stars at the up to date lowest metallicity (Garcia \& Herrero $2013)$. The resulting $\mathrm{T}_{\text {eff }}$ scale is slightly hotter $(\approx 1000 \mathrm{~K})$ than the SMC one.

\section{3. $L M C: 30 \operatorname{Dor}\left(Z=0.50 Z_{\odot}\right)$}

30 Doradus stars were observed in multi-object, multi-epoch spectroscopy mode with FLAMES@VLT, $\mathrm{R}=7000-15000$ and $\lambda=[4000,6800] \AA$ (VFTS project, see Evans et al. 2011). $46 \mathrm{OV}$ and $38 \mathrm{Ovz}$ stars were analyzed with the iacob-gbat tool (SimónDíaz et al. 2011) and the results published in Sabín-Sanjulián et al. (2014). We clarify the nature of OVz stars (O stars having HeII 4686 stronger than both HeII 4542 and HeI 4471), that were suspected to represent the link between early phases of star formation and the normal, slightly evolved, O dwarfs. Sabín-Sanjulián et al. (2014) show that the $\mathrm{OVz}$ phenomenon is a natural consequence of the combination of stellar parameters and that OVz stars may lie away from the Zero Age Main Sequence (ZAMS), although it is easier to find them close to it. Effective temperature and wind strength are the primary stellar parameters controlling the OVz phenomenon, while rotational velocity and surface gravity play a secondary role (Sabín-Sanjulián et al. 2014).

\subsection{Milky Way $\left(Z=1.0 Z_{\odot}\right)$}

We have obtained multi-epoch spectra of 500 Galactic O4-B9 stars with FIES@NOT and HERMES@MERCATOR, with $\mathrm{R} \leqslant 25000$ and $\lambda$ between 3800 and $9000 \AA$. Linebroadening parameters were obtained with the iacob-broad tool (Simón-Díaz \& Herrero 2014), stellar parameters with the iacob-gbat (Simón-Díaz et al. 2011) tool for OB and with the Castro et al. (2012) tool for mid and late B. New results for the distributions of vsini and extra broadening effects in $\mathrm{OB}$ stars have been presented by Simón-Díaz \& Herrero (2014). Among other results, these authors emphasize that a larger number of slowly rotating early B supergiants would be expected as a consequence of angular momentum loss during the evolution of mid O type stars.

\section{A comparison of $\mathbf{T}_{\text {eff }}$ scales}

We found no differences between the $\mathrm{T}_{\text {eff }}$ scales we determined for MW and 30 Dor $\mathrm{O}$ dwarfs, in spite of their metallicity difference. Adding data from the literature does not significantly modify this conclusion. The effect is not seen when comparing $\mathrm{O}$ stars in IC 1613, Sextans A, the SMC and the MW. The most plausible explanation for this behaviour is that we are facing small numbers statistics (Simón-Díaz et al., in prep.).

\section{References}

Castro, N., Urbaneja, M. A., Herrero, A., et al. 2012, A\&A 542, A79

Evans, C. J., Taylor, W. D., Hénault-Brunet, V., et al. 2011, A $\& A$ 530, A108

Garcia, M. \& Herrero, A. 2013, A\&A 551, A74

Herrero, A., Garcia, M., Puls, J., et al. 2012, A\&\&A 543, A85

Kennicutt, R. C. \& Evans, N. J. 2012, ARA\&SA 50, 531

Langer, N. 2012, ARAEA 50, 107

Robertson, B. E., Ellis, R. S., Dunlop, J. S., McLure, R. J., \& Stark, D. P. 2010, Nature 468, 49

Sabín-Sanjulián, C., Simón-Díaz, S., Herrero, A., et al. 2014, A\& A 564, A39

Simón-Díaz, S., Castro, N., Herrero, A., et al. 2011, Journal of Physics Conference Series 328(1), 012021

Simón-Díaz, S. \& Herrero, A. 2014, A\&3A 562, A135 